\title{
Coupling of superconductors through a half-metallic ferromagnet: Evidence for a long-range proximity effect
}

\author{
V. Peña, Z. Sefrioui, D. Arias, C. Leon, and J. Santamaria \\ GFMC Departmento de Fisica Aplicada III, Universidad Complutense de Madrid, 28040 Madrid, Spain \\ M. Varela and S. J. Pennycook \\ Condensed Matter Sciences Division, Oak Ridge National Laboratory, Oak Ridge, Tennessee 37831-6031, USA \\ J. L. Martinez \\ Instituto de Ciencia de Materiales de Madrid (ICMM-CSIC), 28049 Cantoblanco, Madrid, Spain
}

(Received 10 March 2004; published 8 June 2004)

\begin{abstract}
Here we examine the ferromagnetic/superconducting proximity effect in half-metallic ferromagnetic $\mathrm{La}_{0.7} \mathrm{Ca}_{0.3} \mathrm{MnO}_{3}$ and high- $T_{\mathrm{c}}$ superconducting $\mathrm{YBa}_{2} \mathrm{Cu}_{3} \mathrm{O}_{7}$ artificial structures. We have found experimental evidence for the coupling between superconducting layers through ferromagnetic spacers in superlattices. This is consistent with a long-range proximity effect in half-metal ferromagnet/ $d$-wave superconductor structures.
\end{abstract}

DOI: 10.1103/PhysRevB.69.224502

PACS number(s): 74.78.Fk, 74.25.Fy

It is well known that in superconductor $(\mathrm{S}) /$ normal $(\mathrm{N})$ structures superconducting pairing may occur deep into the normal metal. ${ }^{1}$ If the normal metal is a ferromagnet $(\mathrm{F})$, its exchange field reduces drastically the length scale for the proximity effect, ${ }^{2}$ and it should be completely suppressed ${ }^{3}$ in the limiting case of a fully spin polarized ferromagnetic/ singlet superconductor structure. Here we investigate this issue using a high-temperature superconductor $\mathrm{YBa}_{2} \mathrm{Cu}_{3} \mathrm{O}_{7}(\mathrm{YBCO})$ and a spin polarized ferromagnet $\mathrm{La}_{0.7} \mathrm{Ca}_{0.3} \mathrm{MnO}_{3}$ (LCMO). The interplay between magnetism and superconductivity in hybrid structures involving colossal magnetoresistance and high- $T_{\mathrm{c}}$ superconducting oxides has gathered considerable interest in recent years. ${ }^{4}$ Scanning tunneling spectroscopy ${ }^{5}$ and tunneling magnetoresistance ${ }^{6}$ have shown that the LCMO is essentially half metallic (HM). LCMO and YBCO have similar in-plane lattice parameters $(0.3 \%$ mismatch $)$ which allows heteroepitaxial growth with little interface disorder. ${ }^{7-9}$ We find a long-range proximity effect, which yields coupling between superconducting layers through 10-nm thick HM ferromagnetic layers. These $\mathrm{LCMO} / \mathrm{YBCO}$ coupled superlattices represent a class of artificially layered materials showing "coexistence" of spinpolarized ferromagnetism and superconductivity over macroscopic length scales.

In $\mathrm{F} / \mathrm{S}$ structures the transfer of Cooper pairs into the ferromagnet occurs via the Andreev reflection. ${ }^{10}$ Electrons with an energy lower than the superconducting gap are reflected back as holes with opposite spin orientation. The interference between electron and hole wave functions gives rise to the Andreev bound states which carry the supercurrent. Energy conservation requires that Cooper pairs entering a ferromagnet with an exchange field energy $h$ acquire a finite momentum $\Delta p=\eta v_{\mathrm{F}} / h$ where $v_{\mathrm{F}}$ is the Fermi velocity. This causes the superconducting wave function to be oscillating and to decay with a characteristic length scale $\xi_{\mathrm{F}}$ $=(\eta D / 2 \pi h)^{1 / 2}$, where $D$ is the diffusion coefficient. ${ }^{2,11}$ This length is in the nanometer range for common single element or alloy ferromagnets and is typically one to three orders of magnitude smaller than the normal metal coherence length in $\mathrm{N} / \mathrm{S}$ junctions. ${ }^{12,13}$ In a ferromagnet with different number of spin-up $n \uparrow$ and spin-down $n \downarrow$ conduction channels only a fraction $n \downarrow / n \uparrow$ of the majority channels can be Andreev reflected. ${ }^{2}$ Thus, Andreev reflection is completely suppressed for a fully spin polarized ferromagnet (HM) and accordingly the F/S proximity effect, i.e., superconductivity and magnetism should not mix. This is not specific for $s$-wave superconductors but for spin singlet superconductors (either $s$ wave or $d$ wave). In fact, there has been substantial theoretical work in recent years considering the effect of an exchange field in ferromagnet/unconventional ( $d$-wave) superconductor junctions showing that Andreev reflection is suppressed in the limit of a fully spin polarized conduction band. ${ }^{14,15}$

For the present study we have synthesized a number of superconductor (YBCO)/ ferromagnet (LCMO) superlattices and trilayers on (100) oriented STO, using high-pressure (3.4 mbar) pure oxygen sputtering technique at high growth temperature $\left(900^{\circ} \mathrm{C}\right)$. This technique provides a very slow $(0.6 \mathrm{~nm} / \mathrm{min})$ and ordered growth, which allows accurate control of the thickness of the individual layers within one unit cell. Samples were epitaxial and interfaces were atomically flat with little structural disorder. Details about sample preparation and characterization can be found elsewhere. ${ }^{8,9}$ Figure 1 shows a cross-section image of a typical YBCO/ LCMO superlattice obtained in an aberration-corrected HB501UX VG-Microscopes scanning transmission electron microscope. These images show the coherent and ordered growth. Layers are flat and continuous over long lateral distances, as shown in the inset of Fig. 1. For this imaging technique, known as $Z$ contrast, the intensity is roughly proportional to $Z^{2}$, giving direct compositional contrast. Interfaces are perfectly coherent and free of dislocations or defects.

We first present results on F/S/F trilayers. The YBCO thickness was fixed in 12 unit cells $(14 \mathrm{~nm})$ and the LCMO thickness was changed between 2 and 60 unit cells 


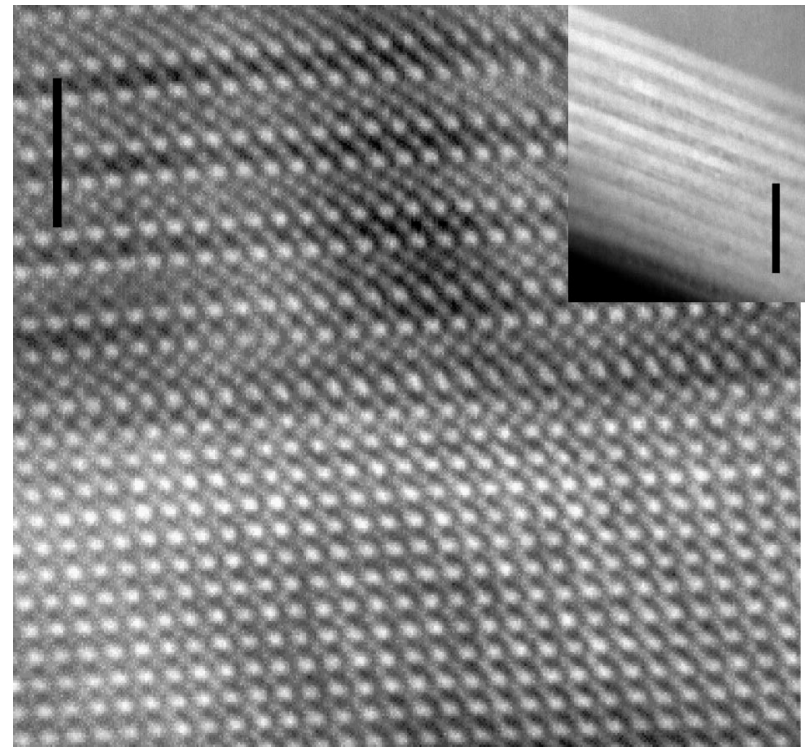

FIG. 1. Z-contrast image of a $\mathrm{YBCO}$ (top)/LCMO(bottom) interface obtained in a aberration corrected HB501UX scanning transmission electron microscope. The scale bar represents $2 \mathrm{~nm}$. Inset: low magnification $Z$-contrast image of a YBCO/LCMO superlattice. The scale bar represents $40 \mathrm{~nm}$.

$(0.8-24 \mathrm{~nm})$. It is important to remark that the thickness of the YBCO has been chosen sufficiently large that the critical temperature is unaffected by dimensionality or epitaxial strain effects. ${ }^{16}$ Samples were magnetic (as measured by superconducting quantum interference device) and superconducting (as measured by transport and susceptibility). Figure 2(a) shows resistance curves of trilayers for different thicknesses of the magnetic layers. Log scale plots (not shown) display sharp and well-behaved superconducting transitions. The superconducting $T_{\mathrm{c}}$ was found to decrease down to a saturation value when the thickness of the magnetic layers was increased [see triangles in Fig. 2(c)]. This result suggests an interplay between magnetism and superconductivity, although a F/S proximity effect cannot be established unambiguously. An alternative mechanism which could cause depression of the critical temperature is pair breaking by injected spin-polarized carriers. ${ }^{17}$ Spin injection is favoured by the $d$-wave character of the superconductivity, where nodes in the superconducting gap along (110) direction open the way to spin-polarized quasiparticle excitations at zero energy cost. To further explore the reason for the depression of the critical temperature in LCMO/YBCO structure we next present results on superlattices with the stacking sequence $\mathrm{F} / \mathrm{S} / \mathrm{F} / \cdots \mathrm{S} / \mathrm{F}$, in which the individual layers have the same thickness as in the F/S/F trilayers. Superlattices had a total thickness of roughly $150 \mathrm{~nm}$, such that the samples with thicker manganite layers had five periods and the samples with the thinnest (three unit cells) manganite layers had ten repetitions.

Within the framework of the F/S proximity effect, the decay of $T_{\mathrm{c}}$ for the trilayers of Fig. 2(c) suggests the order parameter penetrates the LCMO layers up to a thickness $\xi_{\mathrm{F}}$ of $5.2 \mathrm{~nm}$ (as deduced from saturation for trilayers). Superlattices with the described sequence will probe coupling be-
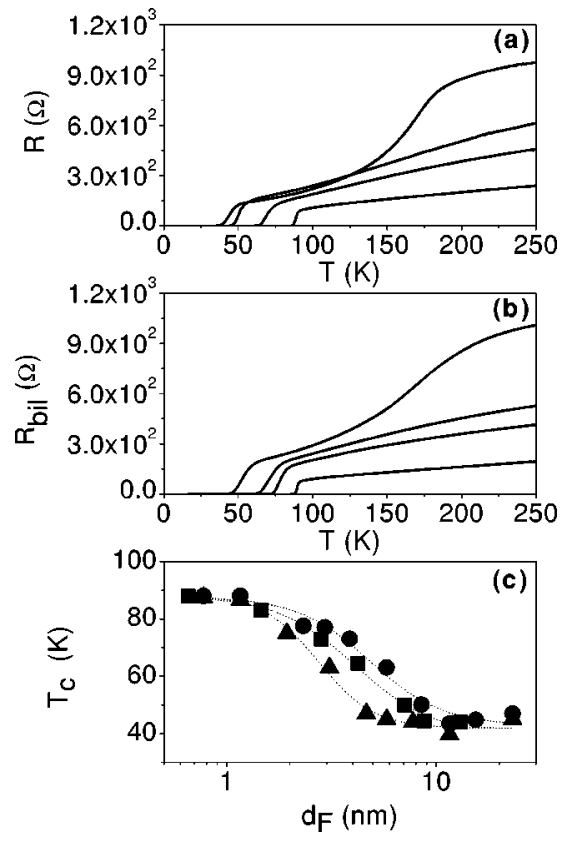

FIG. 2. (a) Resistance vs temperature for [LCMO $\left(N_{\mathrm{M}}\right.$ u.c. $) / \mathrm{YBCO}(12$ u.c. $) / \mathrm{LCMO}\left(N_{\mathrm{M}}\right.$ u.c. $\left.)\right]$ trilayers with $N_{\mathrm{M}}=3,10,15$, and 40 u.c. (b) Resistance vs temperature for [LCMO $\left(N_{\mathrm{M}}\right.$ u.c.)/YBCO (12 u.c.)] superlattices with $N_{\mathrm{M}}=3,10$, 15 , and 40 u.c. Resistance has been normalized to the number of YBCO layers $\left(R_{\mathrm{bil}}\right)$. (c) $T_{\mathrm{c}}$ vs LCMO thickness $d_{\mathrm{F}}$ for $\left[\mathrm{LCMO}\left(N_{\mathrm{M}}\right.\right.$ u.c.)/YBCO $(12$ u.c.) $]$ superlattices (circles) and for [LCMO $\left(N_{\mathrm{M}}\right.$ u.c.)/YBCO $(12$ u.c. $) / \operatorname{LCMO}\left(N_{\mathrm{M}}\right.$ u.c. $\left.)\right]$ trilayers (triangles) and superposition of trilayers [LCMO ( $N_{\mathrm{M}}$ u.c.)/YBCO (12 u.c.)/LCMO $\left(N_{\mathrm{M}}\right.$ u.c.)] (squares). Note that the superpositions of trilayers have magnetic spacers of twice the thickness $\left(2 d_{\mathrm{F}}\right)$ of bottom and top layers $\left(d_{\mathrm{F}}\right)$.

tween the superconducting layers through the magnetic layers for magnetic layer thickness smaller than $2 \xi_{\mathrm{F}}$. If, instead, the depression of the critical temperature were not due to $\mathrm{F} / \mathrm{S}$ (spin injection or any other process occurring in the YBCO) one would expect superlattices to behave exactly the same as trilayers. Figure 2(b) displays resistance curves of a series of superlattices normalized to the number of YBCO layers $\left(R_{\text {bil }}\right.$ in the figure). Again sharp superconducting transitions were found in logarithmic plots (not shown). The depression of the critical temperature with magnetic layer thickness $d_{\mathrm{F}}$ is presented in Fig. 2(c) (circles). One can readily see that saturation occurs at the same value of the critical temperature for trilayers and superlattices. However, throughout the decay, superlattices show higher $T_{\mathrm{c}}$ values for the same LCMO thickness, which provides clear evidence for coupling between the superconducting layers through the HM magnetic layer. Moreover, the characteristic decay length deduced from the saturation LCMO layer thickness is $10.5 \mathrm{~nm}$ which exactly corresponds to $2 \xi_{\mathrm{F}}$, as expected for a $\mathrm{F} / \mathrm{S}$ proximity effect. We stress that this result is independent of mechanisms occurring "inside" the YBCO layers such as spin injection and also on effects arising from interface disorder at length scales shorter than $2 \xi_{\mathrm{F}}$, because such effects are already present in trilayers. Regarding step disorder, one expects it to increase with thickness, resulting, then, in a 
smaller $T_{\mathrm{c}}$ for superlattices than for trilayers, contrary to what is observed. Also, we find both superlattices and trilayers show the same critical temperatures at saturation, implying a negligible effect of interface disorder. In the superlattices experiment we basically probe coupling between superconducting layers, which can only result from the penetration of the superconducting order parameter into the ferromagnet, as expected from a long-range F/S proximity effect. An important remark is that superlattices with magnetic layers of thickness $d_{\mathrm{F}}$ cannot be simply considered as superposition of trilayers each with magnetic layers of thickness $d_{\mathrm{F}} / 2$. To show this we have also grown multilayers which were repetition of trilayers, i.e., bottom and top layers had half the thickness of the ferromagnetic spacers. As in the superlattices, the number of repetitions was chosen to complete $150 \mathrm{~nm}$ total thickness of the samples. The critical temperature dependence on magnetic layer thickness of stacks of trilayers displays a similar steplike behavior with the same saturation value as found for trilayers and superlattices [see squares in Fig. 2(c)]. Stacks of trilayers with thickness $d_{\mathrm{F}}$ of the bottom layer (ferromagnetic spacers of thickness $2 d_{\mathrm{F}}$ ) had $T_{\mathrm{c}}$ values larger than the corresponding single trilayers. In addition, it is smaller than the superlattices with magnetic layers of thickness $d_{\mathrm{F}}$, which is consistent with reduced critical temperatures resulting of reduced coupling through twice thicker magnetic layers.

Additional evidence for coupling between superconductors across a magnetic layer in superlattices can be obtained from hysteresis loops with the magnetic field applied parallel to the layers. We will focus here on two superlattices, one with 15 LCMO unit cells and other with 40 LCMO unit cells spacer thickness (which we will refer to as coupled and decoupled, respectively). Note that the coupled superlattice is at the decaying portion of the $T_{\mathrm{c}}$ vs $d_{\mathrm{F}}$ plot of Fig. 2(c), and the decoupled superlattice is well into saturation, where superlattices and trilayers behave similarly. Figures 3(a) and 3(b) show hysteresis loops above the superconducting transition for decoupled and coupled superlattices, respectively. Saturation magnetization values in excess of $200 \mathrm{emu} / \mathrm{cm}^{3}$ are obtained for both samples, well in the range reported typically for LCMO thin films. The loop corresponding to the thicker LCMO layers [Fig. 3(a)] shows clear steps pointing to coherent magnetization switching within the layers. Figures 3(c) and 3(d) show the corresponding loops below the superconducting transition (at $5 \mathrm{~K}$ ) for both samples. The coupled sample [Fig. 3(d)] displays a characteristic superconductinglike hysteresis loop as expected from coupled YBCO layers across the magnetic layers. The superconducting moment is larger than the ferromagnetic one ( $1.1 \times 10^{-3} \mathrm{emu}$ at saturation for this sample). The central peak is slightly displaced from zero probably due to a small stray field due to the magnetic layers. An estimate of the critical current using Bean model at 1000 Oe (above ferromagnetic saturation) yields $J_{\mathrm{c}}=5.7 \times 10^{4} \mathrm{~A} / \mathrm{cm}^{2}$, smaller than the values obtained for single YBCO films with comparable thickness, typically in excess of $10^{6} \mathrm{~A} / \mathrm{cm}^{2}$. The decoupled sample, on the other hand, shows a ferromagneticlike hysteresis loop [Fig. 3(c)]. This can be understood as resulting from further depression of the superconducting order parameter (critical current), compared to the coupled
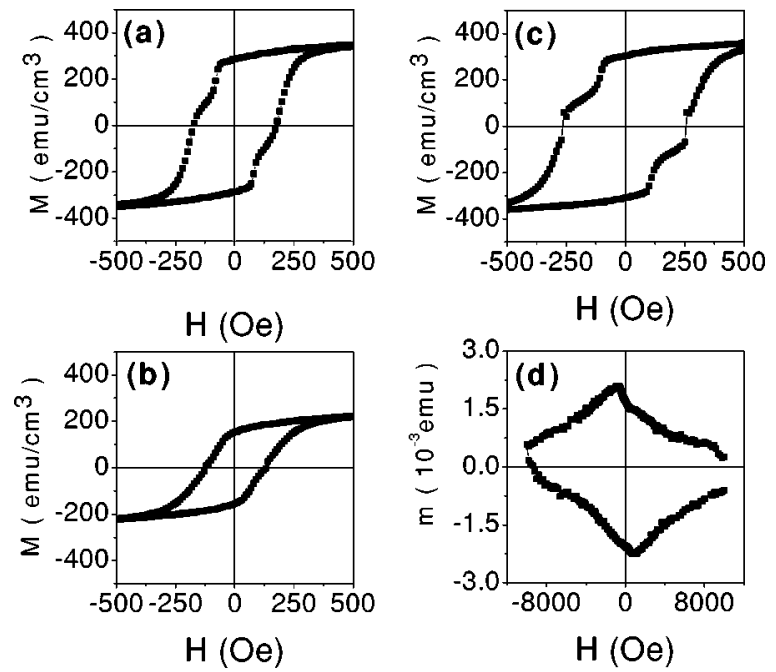

FIG. 3. Hysteresis loops above the superconducting transition for (a) decoupled ( $N_{\mathrm{M}}=40$ u.c.) and (b) coupled (15 u.c.) superlattices measured at $55 \mathrm{~K}$ and $70 \mathrm{~K}$, respectively. Hysteresis loops below the superconducting transition for (c) decoupled $\left(N_{\mathrm{M}}\right.$ $=40$ u.c.) and (d) coupled (15 u.c.) superlattices measured at $5 \mathrm{~K}$.

sample, thus yielding superconducting moments much smaller than in the uncoupled sample. That is, as expected, superconductor coupling through the ferromagnetic layers increases the superconducting magnetic moment.

We thus have provided a strong indication for a longrange $\mathrm{F} / \mathrm{S}$ proximity effect in a $\mathrm{HM}$ ferromagnet. In this respect it is worth mentioning that a long-range proximity effect is consistent with the long-range supercurrents reported for $\mathrm{YBCO} / \mathrm{LCMO} / \mathrm{YBCO}$ junctions years ago. ${ }^{18}$ It is also consistent with the long-range charge transfer from the $\mathrm{YBCO}$ into the LCMO reported recently for $\mathrm{YBCO} / \mathrm{LCMO}$ superlattices from optical measurements. ${ }^{19}$

We now discuss the possible origin of the long-range proximity effect. In ferromagnet/( $d$-wave) superconductor junctions with the interface perpendicular to the $a b$ plane, the transmitted quasiparticles experience different signs of the pairing potential, which results in the formation of zeroenergy bound states (ZES) close to the surface ${ }^{20}$ which are detected as zero-energy peaks in tunneling conductance spectra. In fact, theoretical reports on the tunneling conductance in ferromagnet/ $d$-wave superconductor double tunnel junctions show that ZES originate an enhancement of the quasiparticle tunneling current. ${ }^{21}$ Other ZES mediated processes such as crossed Andreev reflection or elastic cotunneling have been also reported very recently to enhance the conductance of $\mathrm{F} / \mathrm{S} / \mathrm{F}$ junctions. ${ }^{22}$ Although it is clear that ZES may dominate transport properties of $\mathrm{F} / \mathrm{S} / \mathrm{F}$ structures, it is worthwhile to note that in the geometry of our experiment the interface is perpendicular to the $c$ direction and, in principle, transmitted quasiparticles will experience the same sign of the pairing potential and no ZES are expected. ${ }^{23}$ It is therefore not clear how ZES could influence the F/S proximity effect in our samples, especially since there is not a theory for the proximity effect in ferromagnet/ unconventional superconductor junctions.

A different scenario has been theoretically proposed recently which allows proximity effect between a supercon- 
ductor and a half-metallic ferromagnet with domain walls. ${ }^{24}$ According to this work, Cooper-pair-like states may penetrate into the ferromagnet along domain walls, with spin-up and spin-down electrons propagating in neighboring spin-up and spin-down magnetic domains. This requires the domain wall width $D$ being shorter than the in-plane coherence length $[\xi(0) \sim 1.5 \mathrm{~nm}]$. For the thickness of the magnetic layers used in this work $D$ can be estimated to be in the range $10-20 \mathrm{~nm}$, longer than $\xi(0)$. However, close to $T_{\mathrm{c}}, \xi(T)$ can be much longer $\left[\xi(T)=\xi(0) /\left(1-T / T_{c}\right)^{1 / 2}\right]$ and it is not unrealistic to think that this mechanism could justify a proximity effect in our samples.

Finally, another possibility we want to discuss is related to recent theoretical predictions ${ }^{25-27}$ of a long-range F/S proximity effect resulting of triplet correlations appearing at the interface between a half-metallic ferromagnet and a superconductor. Evidences for triplet superconductivity have been recently found for $\mathrm{Sr}_{2} \mathrm{RuO}_{4}$ (Ref. 28) and heavy-fermion materials. ${ }^{29,30}$ Triplets are not sensitive to the exchange field and the coexistence of ferromagnetism and superconductivity becomes possible, as reported for URhGe (Ref. 29) or $\mathrm{ZrZn}_{2} \cdot{ }^{30} \mathrm{~A}$ triplet condensate coexisting with conventional singlet pairing but decaying slowly into a ferromagnet has been recently proposed in a superconductor/ferromagnet junction arising as a result of nonhomogeneous magnetization at the interface ${ }^{25,26}$ or from reflection of singlet pairs at the HM interface. ${ }^{27}$ However, triplet superconductivity is still considered a rare low-temperature phenomenon and its occurrence in the F/S structures described here will require further work.

To conclude, our results provide evidence for coupling between superconducting layers through ferromagnetic spacers consistent with a long-range F/S proximity effect. This result may be stimulating for the development of a theory of the F/S proximity effect between an unconventional superconductor and a half-metallic ferromagnet. In addition, these results may provide important clues for the coexistence between magnetism and superconductivity in oxide systems like $\mathrm{RuSr}_{2} \mathrm{GdCu}_{2} \mathrm{O}_{8}$ (Ref. 31) where clearly distinguishable subsystems support either ferromagnetism or superconductivity.

This work was supported by MCYT MAT 2002-2642 and CAM 07N/0032/2002 and Fundación Ramón Areces.
${ }^{1}$ For a review see C. W. J. Beenakker, Rev. Mod. Phys. 69, 731 (1997).

${ }^{2}$ Z. Radovic, L. Dobrosavljevic-Grujic, A. I. Buzdin, and J. R. Clem, Phys. Rev. B 38, 2388 (1988).

${ }^{3}$ M. J. M. de Jong and C. W. J. Beenakker, Phys. Rev. Lett. 74, 1657 (1995).

${ }^{4}$ C. A. R. Sá de Melo, Phys. Rev. Lett. 79, 1933 (1997); Phys. Rev. B 62, 12303 (2000).

${ }^{5}$ J. Y. T. Wei, N. -C. Yeh, and R. P. Vasquez, Phys. Rev. Lett. 79, 5150 (1997).

${ }^{6}$ Moon-Ho Jo, N. D. Mathur, N. K. Todd, and M. G. Blamire, Phys. Rev. B 61, R14905 (2000).

${ }^{7}$ H.-U. Habermeier, G. Cristiani, R. K. Kremer, O. Lebedev, and G. van Tendeloo, Physica C 364, 298 (2001).

${ }^{8}$ Z. Sefrioui, M. Varela, V. Peña, D. Arias, C. León, J. Santamaria, J. E. Villegas, J. L. Martinez, W. Saldarriaga, and P. Prieto, Appl. Phys. Lett. 81, 4568 (2002).

${ }^{9}$ Z. Sefrioui, D. Arias, V. Peña, J. E. Villegas, M. Varela, P. Prieto, C. León, J. L. Martínez, and J. Santamaria, Phys. Rev. B 67, 214511 (2003).

10 A. F. Andreev, Zh. Eksp. Teor. Fiz. 46, 1823 (1964) [Sov. Phys. JETP 19, 1228 (1964)].

${ }^{11}$ E. A. Demler, G. B. Arnold, and M. R. Beasley, Phys. Rev. B 55, 15174 (1997).

${ }^{12}$ L. V. Mercaldo, C. Attanasio, C. Coccorese, L. Maritato, S. L. Prischepa, and M. Salvato, Phys. Rev. B 53, 14040 (1996).

${ }^{13}$ J. Aarts and J. M. E. Geers, Phys. Rev. B 56, 2779 (1997).

${ }^{14}$ J. X. Zhu, B. Friedman, and C. S. Ting, Phys. Rev. B 59, 9558 (1999).

${ }^{15}$ S. Kashiwaya, Y. Tanaka, N. Yoshida, and M. R. Beasley, Phys. Rev. B 60, 3572 (1999).

${ }^{16}$ M. Varela, Z. Sefrioui, D. Arias, M. A. Navacerrada, M. Lucía, M. A. López de la Torre, C. León, G. D. Loos, F. Sánchez-
Quesada, and J. Santamaría, Phys. Rev. Lett. 83, 3936 (1999).

${ }^{17}$ N.-C. Yeh, R. P. Vasquez, C. C. Fu, A. V. Samoilov, Y. Li, and K. Vakili, Phys. Rev. B 60, 10522 (1999).

${ }^{18}$ M. Kasai, Y. Kanke, T. Ohno, and Y. Kozono, J. Appl. Phys. 72, 5344 (1992); Y. Kanke, M. Kasai, T. Ohno, and Y. Kozono, ibid. 74, 2681 (1993).

${ }^{19}$ Todd Holden, H.-U. Habermeier, G. Cristiani, A. Golnik, A. Boris, A. Pimenov, J. Humlícek, O. I. Lebedev, G. Van Tendeloo, B. Keimer, and C. Bernhard, Phys. Rev. B 69, 064505 (2004).

${ }^{20}$ C. R. Hu, Phys. Rev. Lett. 72, 1526 (1994).

${ }^{21}$ N. Yoshida, Y. Tanaka, J. Inoue, and S. Kashiwaya, Phys. Rev. B 63, 024509 (2000).

${ }^{22}$ N. Stefanakis and R. Melin, J. Phys.: Condens. Matter 15, 4239 (2003).

${ }^{23}$ Y. Tanaka and S. Kashiwaya, Phys. Rev. Lett. 74, 3451 (1995).

${ }^{24}$ R. Melin and S. Peysson, Phys. Rev. B 68, 174515 (2003).

${ }^{25}$ F. S. Bergeret, A. F. Volkov, and K. B. Efetov, Phys. Rev. Lett. 86, 4096 (2001).

${ }^{26}$ A. F. Volkov, F. S. Bergeret, and K. B. Efetov, Phys. Rev. Lett. 90, 117006 (2003).

${ }^{27}$ M. Eschrig, J. Kopu, J. C. Cuevas, and Gerd Schön, Phys. Rev. Lett. 90, 137003 (2003).

${ }^{28}$ K. Ishida, H. Mukuda, Y. Kitaoka, K. Asayama, Z. Q. Mao, Y. Mori, and Y. Maeno, Nature (London) 396, 658 (1998).

${ }^{29}$ D. Aoki, A. Huxley, E. Ressouche, D. Braithwaite, J. Flouquet, J. P. Brison, E. Lhotel, and C. Paulsen, Nature (London) 413, 613 (2001).

${ }^{30}$ C. Pfleiderer, M. Uhlarz, S. M. Hayden, R. Vollmer, H. von Lohneysen, N. R. Bernhoeft, and G. G. Lonzarich, Nature (London) 412, 58 (2001).

${ }^{31}$ I. Felner, U. Asaf, Y. Levi, and O. Millo, Phys. Rev. B 55, R3374 (1997). 